\title{
Positive-unlabelled learning of glycosylation sites in the human proteome
}

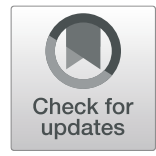

Fuyi Li ${ }^{1,2}$, Yang Zhang ${ }^{3}$, Anthony W. Purcell', Geoffrey I. Webb ${ }^{2}$, Kuo-Chen Chou ${ }^{4,5}$, Trevor Lithgow ${ }^{6}$, Chen $\mathrm{Li}^{1,7^{*}}$ and Jiangning Song ${ }^{1,2^{*}}$ (D)

\begin{abstract}
Background: As an important type of post-translational modification (PTM), protein glycosylation plays a crucial role in protein stability and protein function. The abundance and ubiquity of protein glycosylation across three domains of life involving Eukarya, Bacteria and Archaea demonstrate its roles in regulating a variety of signalling and metabolic pathways. Mutations on and in the proximity of glycosylation sites are highly associated with human diseases. Accordingly, accurate prediction of glycosylation can complement laboratory-based methods and greatly benefit experimental efforts for characterization and understanding of functional roles of glycosylation. For this purpose, a number of supervised-learning approaches have been proposed to identify glycosylation sites, demonstrating a promising predictive performance. To train a conventional supervised-learning model, both reliable positive and negative samples are required. However, in practice, a large portion of negative samples (i.e. non-glycosylation sites) are mislabelled due to the limitation of current experimental technologies. Moreover, supervised algorithms often fail to take advantage of large volumes of unlabelled data, which can aid in model learning in conjunction with positive samples (i.e. experimentally verified glycosylation sites).

Results: In this study, we propose a positive unlabelled (PU) learning-based method, PA2DE (V2.0), based on the AlphaMax algorithm for protein glycosylation site prediction. The predictive performance of this proposed method was evaluated by a range of glycosylation data collected over a ten-year period based on an interval of three years. Experiments using both benchmarking and independent tests show that our method outperformed the representative supervised-learning algorithms (including support vector machines and random forests) and oneclass learners, as well as currently available prediction methods in terms of F1 score, accuracy and AUC measures. In addition, we developed an online web server as an implementation of the optimized model (available at http:// glycomine.erc.monash.edu/Lab/GlycoMine_PU/) to facilitate community-wide efforts for accurate prediction of protein glycosylation sites.

Conclusion: The proposed PU learning approach achieved a competitive predictive performance compared with currently available methods. This PU learning schema may also be effectively employed and applied to address the prediction problems of other important types of protein PTM site and functional sites.
\end{abstract}

Keywords: Protein glycosylation prediction, Positive unlabelled-learning, Supervised-learning, AlphaMax, Sequence analysis, Sequence-derived features

\footnotetext{
* Correspondence: Chen.Li@monash.edu; Jiangning.Song@monash.edu ${ }^{1}$ Infection and Immunity Program, Biomedicine Discovery Institute and Department of Biochemistry and Molecular Biology, Monash University, Melbourne, VIC 3800, Australia

Full list of author information is available at the end of the article
}

(c) The Author(s). 2019 Open Access This article is distributed under the terms of the Creative Commons Attribution 4.0 International License (http://creativecommons.org/licenses/by/4.0/), which permits unrestricted use, distribution, and reproduction in any medium, provided you give appropriate credit to the original author(s) and the source, provide a link to the Creative Commons license, and indicate if changes were made. The Creative Commons Public Domain Dedication waiver (http://creativecommons.org/publicdomain/zero/1.0/) applies to the data made available in this article, unless otherwise stated. 


\section{Background}

Glycosylation is among the most ubiquitous and important type of post-translational modification (PTM) across three domains of life, including Eukarya, Bacteria, and Archaea [1]. It is estimated that glycosylation may occur in $>50 \%$ of the human proteins [2], and that it is ubiquitous in all living organisms [3]. Glycosylation involves attachment of different types of glycan molecules to a specific amino acid side-chain (i.e., tryptophan, asparagine, serine, or threonine) in protein substrates [4]. Glycosylation has been reported to be relevant for a myriad of biological processes, including cell signalling and communication, cell dissociation, immune modulation, protein quality control, protein folding, subcellular localization, and degradation [5-12]. Based on its critical role in a wide variety of major pathways, protein glycosylation is associated with a variety of human diseases, including diabetes [13-15], cancers [16-20], and autoimmune diseases [21-23]. In light of these strong associations with human diseases, and in the current era of precision medicine, there is an urgent need to develop computational tools to accurately predict glycosylation sites in order to prioritize potential candidates for experimental validation and elucidate their biological functions.

To shortlist potential glycosylation sites and facilitate advanced experimental validation, a variety of computational methods have been proposed as useful alternative approaches. Such in silico methods for glycosylation site prediction include NetNGlyc [24], NetOGlyc [21], EnsembleGly [25], GPP [26], GlycEP [27], ModPred [28], as well as our previously developed tools GlycoMine [29] and GlycoMine $^{\text {struct }}$ [30], etc. These approaches are based on a supervised-learning scheme [e.g. using supervised learning algorithms such as support vector machines (SVMs), random forest (RF), etc] that uses reliably labelled positive (i.e. experimentally verified glycosylation sites) and negative (i.e. non-glycosylation sites) samples to train the prediction model. In terms of negative sample selection, the majority of current approaches, such as NetNGlyc, NetOGlyc, EnsembleGly, GPP, GlycEP, and GlycoMine ${ }^{\text {Struct }}$ randomly selected non-glycosylation sites from experimentally verified glycosylated proteins as the negative samples. However, this strategy can be problematic, as previously assigned negative samples (non-glycosylation sites) could be mislabelled due to limitations in experimental conditions and technologies used, potentially resulting in unreliable negative data selection and biased model training. Moreover, most current methods fail to account for the vast amount of unlabelled data, the majority of which have not been annotated with respect to glycosylation. According to a previous study by De Comite et al. [31], positive and unlabelled samples can aid the learning process. Recently, Niu et al. [32] demonstrated theoretically that positive unlabelled (PU)-learning methods performed better than supervised learning on PU scenarios. Another two recent works highlighted that PU learning can yield an equivalent performance to supervised-learning algorithms [33, 34] when using ranking-based performance measures, such as receiver operating characteristic (ROC) curve and the precision-recall curve. Most recently, a powerful bioinformatics tool, MutPred2 [35], has applied the PU learning approaches to address the problem of inferring the molecular and phenotypic impact of amino acid variants, and achieved a favourable performance compared with supervised-learning algorithms. These studies demonstrate that PU learning has a great capacity to achieve at least competitive performance compared to supervised-learning algorithms and thus effectively avoid the labour-intensive data labelling procedure. Motivated by these studies, in this study, we employed a PU-learning scheme to utilize the vast amount of unlabelled data in order to explore the possibility of achieving a competitive performance compared to the traditional supervised-learning approaches with more relaxed requirement for data labelling.

For the current task, our results suggested that the advantages of PU learning relative to traditional supervised-learning techniques can be summarized as follows: 1) PU learning is fast and simple, is able to significantly reduce the effort and time necessary to label samples and can achieve a competitive performance compared to supervised-learning algorithms [36-38]; and 2) PU-learning is particularly amenable to bioinformatics and computational biology settings, where a sizable portion of previously unidentified samples is likely mislabelled.

In this study, we proposed a novel method, PA2DE (V2.0), under the PU learning scenario for glycosylation sites prediction. We also benchmarked several state-of-the-art PU-learning algorithms and compared the performance of our method with these algorithms for glycosylation prediction using time-scaled datasets collected between 2007 and 2016 and sequence-derived features. The predictive performance of the proposed method was extensively benchmarked against state-of-the-art PU-learning algorithms, traditional supervised-learning algorithms (i.e. SVMs and RFs) and one-class classifiers on both benchmark and independent test datasets. The results showed that PA2DE (V2.0) achieved an outstanding predictive performance in terms of $\mathrm{F} 1$ score, accuracy (ACC), and the area under the curve (AUC) values. Next, we retrained the classifiers of PA2DE (V2.0) using a more comprehensive dataset and further compared its predictive performance with several state-of-the-art glycosylation site prediction methods. The performance comparison results demonstrated that PA2DE (V2.0) achieved a competitive performance compared with these methods. Finally, we developed an online web server as an implementation of the proposed method to facilitate the community-wide efforts for performing in silico glycosylation site prediction. 


\section{Results}

\section{Overall framework}

Figure 1 illustrates the generic framework used for benchmarking the performance of our proposed method with PU-learning, supervised learning and one-class classification algorithms for glycosylation prediction. As can be shown, this framework comprised three steps, including data collection and pre-processing, feature extraction and selection, and benchmarking and independent tests. At the first step, four time-scaling datasets harbouring experimentally verified $\mathrm{C}$-, $\mathrm{N}$-, and O-linked human glycosylation sites collected in 2007, 2010, 2013, and 2016, respectively, were extracted from the UniProt database [39]. We subsequently performed sequence homology reduction in order to remove the sequence redundancy from the initial datasets. At the second step, a variety of sequence-derived features were calculated and extracted from all four datasets. Feature selection based on the

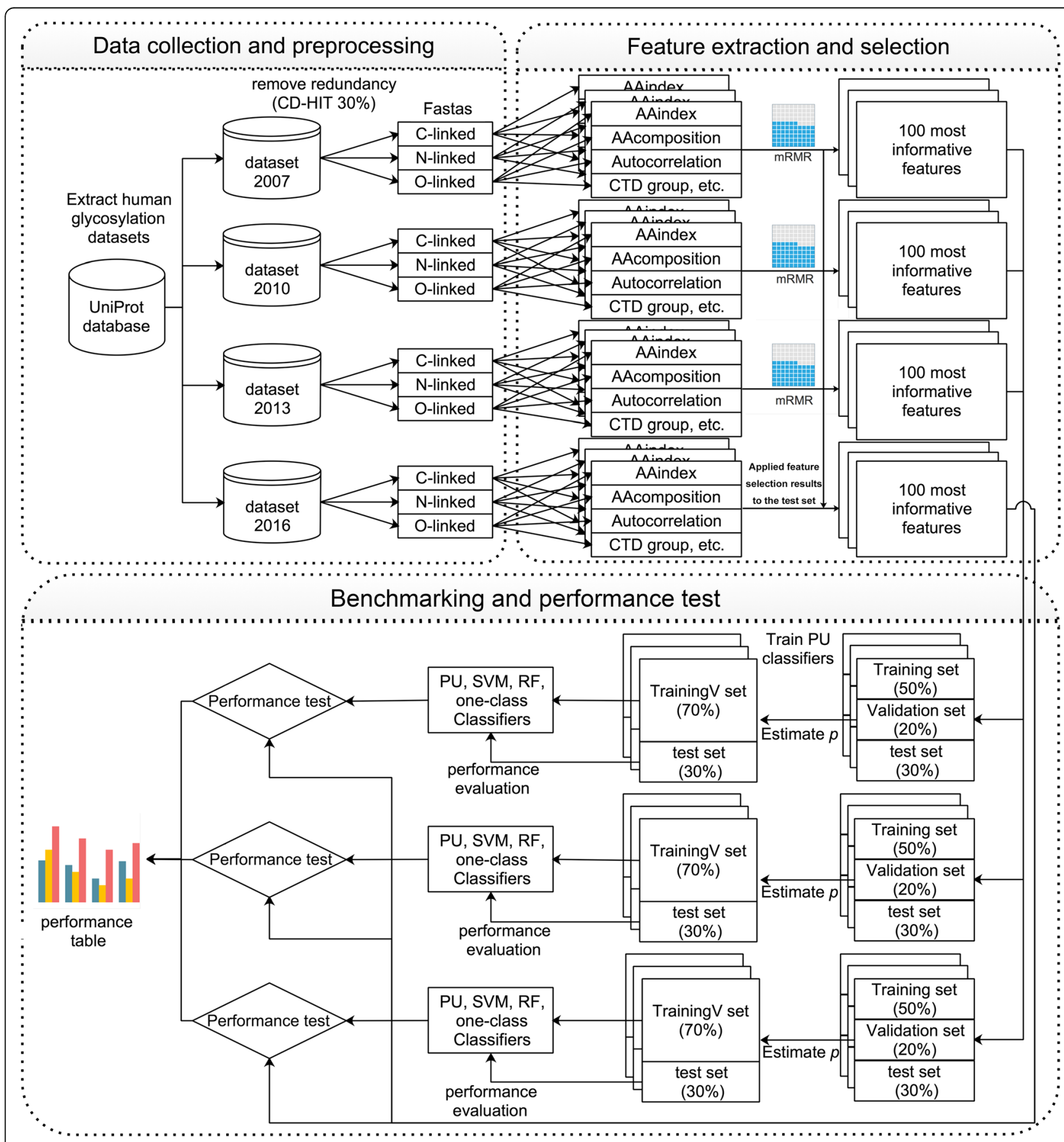

Fig. 1 The overall framework of the experiments 
maximal Redundancy Maximal Relevance (mRMR) [40] algorithm was then conducted to eliminate the redundant and irrelevant features. As a result, the top 100 features were ranked and identified for the datasets comprising C-, $\mathrm{N}-$, and O-linked glycosylation data for each year (Refer to the section "Feature extraction and selection"). Please note that the feature selection was only conducted for training sets (i.e. datasets extracted from 2007, 2010, and 2013) and the selection results (i.e. the selected features) were then applied to the test set (i.e. the dataset extracted from 2016). At the final step, we performed the benchmarking and performance tests using these datasets and the correspondingly selected features. Three types of prediction models trained using PU-learning, supervised-learning, and one-class classification algorithms were constructed, evaluated, and compared.

\section{Dataset analysis}

We collected four experimentally verified human C-, N-, and O-linked glycosylation site datasets in the years 2007, 2010, 2013, and 2016 from the UniProt database. A statistical summary of the collected proteins along with their glycosylation sites before and after the sequence-redundancy removal is shown in Table 1.

We first analysed the number of previously mislabelled negative samples (i.e. non-glycosylation sites) in data collected over four years (2007, 2010, 2013, and 2016). The detailed numbers highlighting the previously mislabelled negative sites are shown in Table 2.

In Table 2, the N1 rows of the years 2010, 2013, and 2016 show the numbers of mislabelled non-glycosylation sites and the percentages as compared with those of the corresponding previous collection years (i.e. 2007, 2010, and 2013) for C-, N-, and O-linked glycosylation, respectively. For example, the $\mathrm{N} 1$ value of $\mathrm{N}$-linked glycosylation in
2010 was 237, which means that there existed 237 sites, which were labelled as non-glycosylation sites in 2007 but were later labelled as N-linked glycosylation sites in 2010 . These 237 mislabelled sites accounted for $26.04 \%$ of all newly added sites in 2010 compared with 2007 (e.g. 26.04\% $=237 /(2118-1208)$, where 2118 was the number of N-linked glycosylation sites in 2010, while 1208 was the number of N-linked glycosylation sites in 2007). As shown in Table 2, a significant number of non-glycosylation sites were labelled incorrectly due to the limitations of experimental technologies, suggesting the possibility that current non-glycosylation sites might actually represent true positives. With the advances of new technologies, additional previously labelled non-glycosylation sites will likely also become true positives. Importantly, this issue also applies to other typical bioinformatics problems, such as other types of PTMs (such as phosphorylation [41], lysine PTMs [42], cleavage sites [43-45] etc.) and protein-protein interaction prediction [46], for which the selection of negative samples should be exercised with caution. This issue also highlights the significance of using PU-learning algorithms to address such tasks and employing only positive and unlabelled samples to train the models.

While the P1 rows are the actual probability of the glycosylation sites of the previous collection time point. For example, the P1 value of N-linked glycosylation in 2010 was $3.38 \%$, meaning that the number of the positive samples accounts for $3.38 \%$ of the total number of samples $(3.38 \%=(237+1208) /(41,526+1208)$, where 237 is the number of mislabelled non-glycosylation sites in the 2007 dataset, 1208 is the number of glycosylation sites in the 2007 dataset, and 41,526 is the total number of unlabelled sites). The P2 rows are the prior probabilities of the glycosylation sites estimated by the Elkan-Noto algorithm, while the P3 rows are the prior probabilities of

Table 1 A statistical summary of glycosylated proteins and glycosylation sites collected from 2007, 2010, 2013, and 2016 data

\begin{tabular}{|c|c|c|c|c|c|}
\hline \multirow[t]{2}{*}{ Year } & \multirow[t]{2}{*}{ Type } & \multicolumn{2}{|c|}{ Initial dataset prior to redundancy removal } & \multicolumn{2}{|c|}{ Final dataset after redundancy removal } \\
\hline & & Num. of sites & Num. of substrates & Num. of sites & Num. of substrates \\
\hline \multirow[t]{3}{*}{2007} & C-linked & 36 & 10 & 36 & 10 \\
\hline & N-linked & 1245 & 537 & 1208 & 520 \\
\hline & O-linked & 321 & 101 & 320 & 100 \\
\hline \multirow[t]{3}{*}{2010} & C-linked & 38 & 12 & 38 & 12 \\
\hline & N-linked & 2175 & 908 & 2118 & 872 \\
\hline & O-linked & 345 & 114 & 344 & 113 \\
\hline \multirow[t]{3}{*}{2013} & C-linked & 43 & 15 & 43 & 15 \\
\hline & N-linked & 2508 & 1004 & 2442 & 965 \\
\hline & O-linked & 474 & 178 & 455 & 162 \\
\hline \multirow[t]{3}{*}{2016} & C-linked & 46 & 17 & 46 & 17 \\
\hline & N-linked & 2805 & 1111 & 2728 & 1066 \\
\hline & O-linked & 698 & 221 & 679 & 212 \\
\hline
\end{tabular}


Table 2 Summary of the results for mislabelled negative sites

\begin{tabular}{lllll}
\hline Year & & C-linked & N-linked & O-linked \\
\hline 2010 & N1 & 0 & $237(26.04 \%)$ & $22(91.67 \%)$ \\
& P1 & $11.76 \%$ & $3.38 \%$ & $1.26 \%$ \\
& P2 & $14.86 \%$ & $3.41 \%$ & $1.28 \%$ \\
& P3 & $12.07 \%$ & $3.39 \%$ & $1.28 \%$ \\
& N1 & 0 & $119(36.73 \%)$ & $32(19.82 \%)$ \\
& P1 & $8.35 \%$ & $3.01 \%$ & $1.22 \%$ \\
& P2 & $9.36 \%$ & $4.36 \%$ & $1.24 \%$ \\
& P3 & $8.62 \%$ & $3.97 \%$ & $1.22 \%$ \\
& N1 & 0 & $99(34.62 \%)$ & $32(19.82 \%)$ \\
& P1 & $6.51 \%$ & $3.09 \%$ & $1.11 \%$ \\
& P2 & $7.15 \%$ & $4.68 \%$ & $1.13 \%$ \\
& P3 & $6.63 \%$ & $3.83 \%$ & $1.13 \%$ \\
\hline
\end{tabular}

Note: a) N1, numbers and percentages of mislabelled non-glycosylation sites and their percentages as compared with previous collection years; b) P1, the actual class probability of glycosylation sites; c) P2: the prior probability of glycosylation sites estimated by the Elkan-Noto algorithm; d) P3: the prior probability of glycosylation sites estimated by the AlphaMax algorithm

the glycosylation sites estimated by the AlphaMax algorithm. In general, P3 and P2 are similar with the P1, but they are both relatively higher than P1. In addition, the value of P3 is closer to P1 than P2, which indicates the AlphaMax algorithm is more reliable than the
Elkan-Noto algorithm in terms of prior probability estimation.

We further analysed the proportions of unlabelled samples. Note that the unlabelled samples included non-glycosylation sites and potential glycosylation sites yet to be discovered. Based on the data shown in Table 1, we generated Fig. 2 to visually illustrate the large amounts of unlabelled samples of C-, N-, and O-linked glycosylation in chronological order from 2007 to 2016. The bar charts in Fig. 2 show the number of glycosylation sites identified each year based on Table 1, whereas the pie charts illustrate the percentage of glycosylation sites relative to unlabelled sites and associated with $\mathrm{C}$-, $\mathrm{N}$-, and O-linked glycosylation, respectively.

With the development of more advanced experimental technologies, increasing numbers of glycosylated proteins and corresponding glycosylation sites are being characterized (Fig. 2), with more unlabelled samples also available. For example, $1208 \mathrm{~N}$-linked glycosylation sites were identified in 2007, accounting for $8 \%$ of the total number of potential N-linked glycosylation sites. This number increased to 2118 and 2442 in 2010 and 2013, respectively, whereas the percentages remained at $7 \%$. In the case of C-linked glycosylation, a significant increase from 71 to $84 \%$ in the proportion of unlabelled samples occurred from 2007 to 2016. Altogether, these data

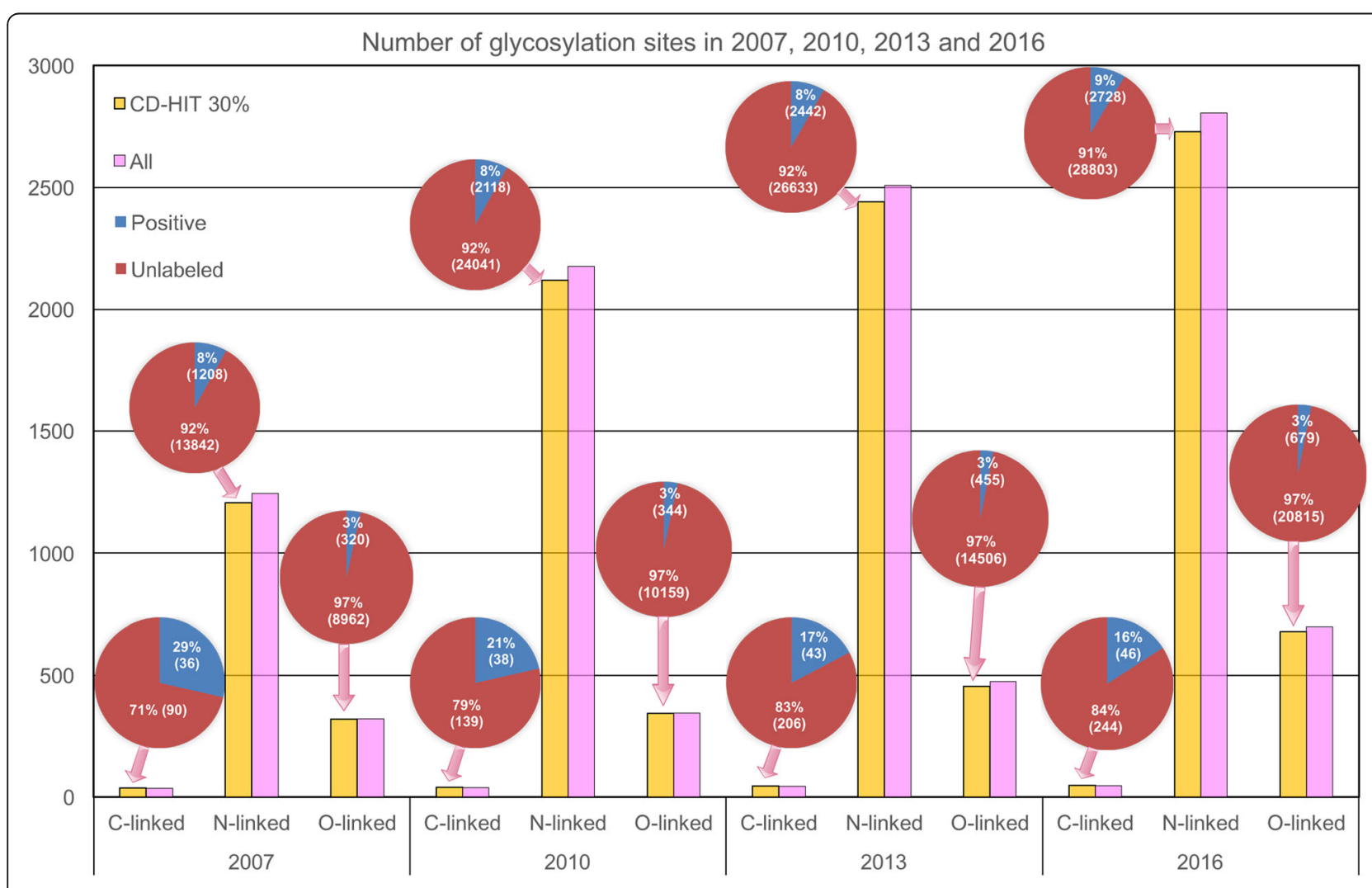

Fig. 2 Rapid increase in the numbers of glycosylation sites and unlabelled samples in an increasing chronological order (from years 2007 to 2016) 
associated with mislabelled non-glycosylation sites and the increasing number of unlabelled sites motivated us to explore the possibility of employing the PU-learning algorithms to facilitate the prediction of glycosylation sites by considering unlabelled sites.

\section{Performance comparison of different algorithms on the benchmark datasets}

We evaluated and compared the predictive performance of the PU-learning, supervised-learning, and one-class classification algorithms on the benchmark datasets of $\mathrm{C}-, \mathrm{N}-$, and O-linked glycosylation collected from 2007, 2010, and 2013. Based on each dataset, we performed 100 randomization tests and reported the averaged performance results. For each experiment, the same numbers of unlabelled and positive samples were selected to form an initial dataset, which was further randomly split into three subsets: training set $(50 \%)$, validation set (20\%), and test set (30\%). The validation set was used to estimate the prior probability of the positive class [36], and the training and validation sets were combined as a new training set (i.e. trainingV; Fig. 1) to retrain the classifiers. The predictive performance of the trained classifiers was evaluated using the test set, and the average performance results from 100 experiments were reported. Note that for the supervised-learning algorithms (i.e. SVM and RF), the unlabelled sites were regarded as negative samples, and we directly used the training sets to train the supervised-learning classifiers. For the one-class classification algorithms, we only used positive samples from the training sets to train the algorithms.

The average predictive performance (measured by F1, ACC, and AUC) of the classifiers on the benchmark datasets is shown in Table 3. The best F1, ACC, and AUC values for each experiment are underlined and marked in bold. These results showed that the PU-learning algorithms generally outperformed the supervised-learning and one-class classification algorithms in terms of F1, ACC, and AUC, with the only exception for the dataset of O-linked glycosylation from 2013. For the PU-learning algorithms, PA2DE, PTAN, and PNB performed best in most cases.

PU-learning algorithms performed best on the test datasets To objectively compare the predictive performances, we conducted performance tests of all of the algorithms using the samples included in the dataset from 2016 rather than those from 2013, 2010, and 2007 as the positive test dataset. The numbers of tested positive samples are shown in Table 4 . We only evaluated the performance of $\mathrm{N}$-linked and O-linked glycosylation sites, due to the limited availability of C-linked data (only three C-linked glycosylation sites). We then randomly chose negative samples that were not labelled as glycosylation sites in all of the datasets across all the four years. This process was repeated 100 times, resulting in 100 test datasets incorporating positive datasets and different randomly selected negative datasets. We applied these 100 test datasets to evaluate the classifiers used in the benchmark test. The average predictive performance in terms of F1, ACC, and AUC are reported in Table 5.

The predictive performance of the algorithms on the test datasets showed that PA2DE (V2.0) performed best for both $\mathrm{N}$ - and O-linked glycosylation site prediction in terms of F1 and ACC. Additionally, PNB achieved the highest AUC values on the O-linked datasets of 2007 and 2013. On the N-linked dataset of 2010, PFBC achieved the best AUC value, while RF achieved the best AUC value when trained using the $2007 \mathrm{~N}$-linked glycosylation datasets, while SVM achieved the best ACC value on the O-linked dataset of 2010. Compared to the PU-learning and supervised-learning algorithms, the one-class learners performed the worst across all these years in terms of AUC and accuracy.

As shown in Table 5, in most cases, PA2DE (V2.0) performed best among the PU-learning algorithms on the test datasets. To examine the statistical significance of F1 improvement by PA2DE (V2.0), we performed a Student's $t$-test to compare the results from PA2DE (V2.0), PA2DE, RF, and SVM. Table 6 provides the calculated $p$-values, which indicate that the F1 of PA2DE was significantly $(p \leq 0.01)$ higher than that for RF and SVM according to eight pairwise tests (marked in bold) among a total of 12 tests. Figure 3 plots the distributions of F1 scores for these algorithms on the test datasets, with the average F1 scores for PA2DE substantially higher than that for RF and SVM.

\section{Comparison with existing methods and web server implementation}

Thus far, we have used four time-scaling datasets collected from the UniProt database to compare the prediction performance of PA2DE (V2.0) with PU-learning, supervised learning and one-class classification algorithms. The results demonstrated that the PA2DE (V2.0) algorithm achieved the best performance in most scenarios.

In this section, we constructed a complete dataset with experimentally validated human glycosylation sites collected from the UniProt, dbPTM [47], and PhosphoSitePlus [48] databases to make the performance comparison with the existing methods. For the data extracted from the UniProt database, we only considered glycosylation sites with ECO code ECO:0000269, which indicates the manually curated information with published experimentally evidence (https://www.uniprot.org/help/evidences). We further implemented an online web server using an optimized PA2DE (V2.0) algorithm trained on this new dataset. According to a previous study [28], N-linked 


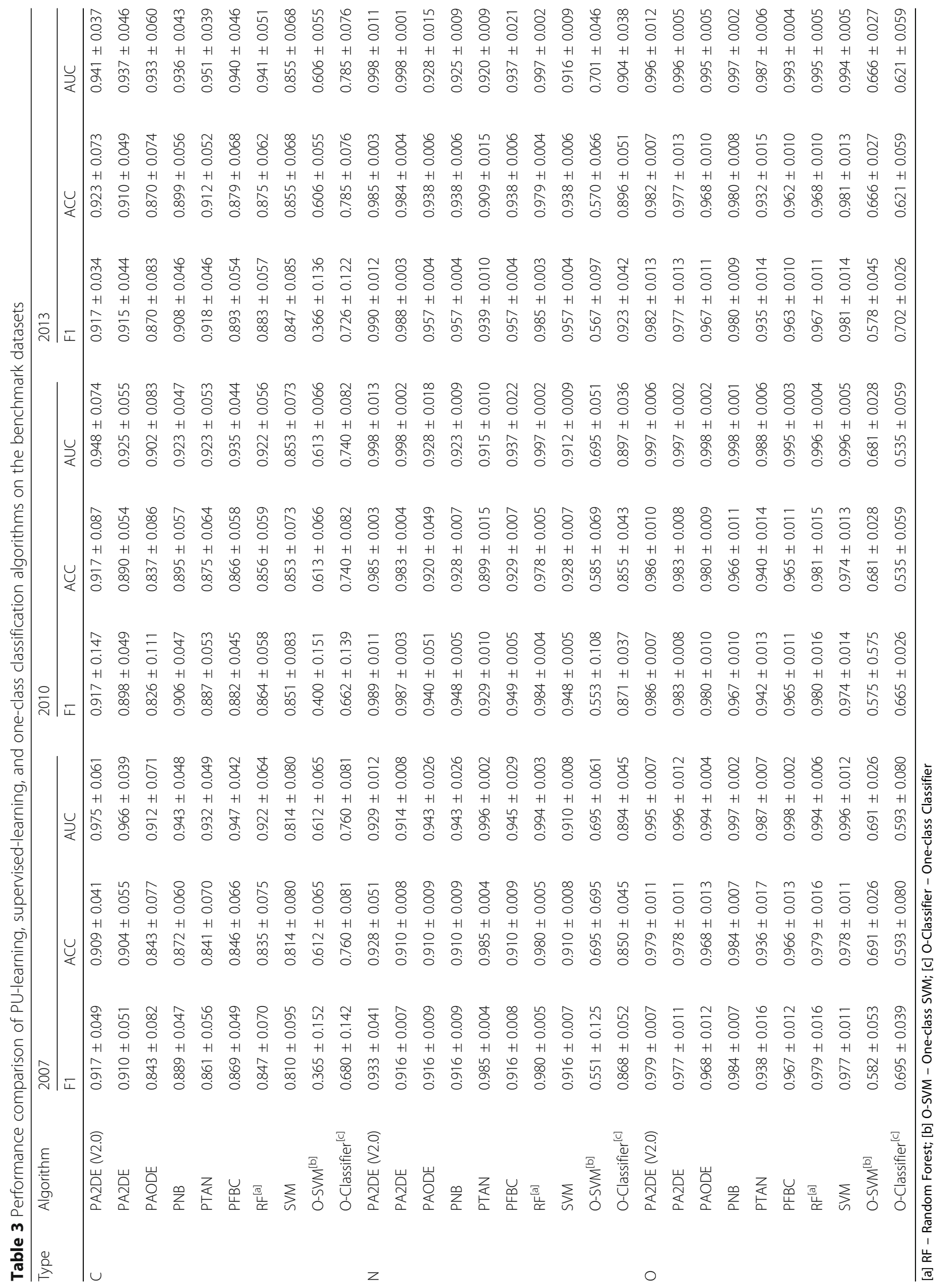


Table 4 The numbers of glycosylated proteins and corresponding sites included in the test datasets

\begin{tabular}{lll}
\hline Type & Num. of Sites & Num. of Substrates \\
\hline C-linked & 3 & 2 \\
N-linked & 324 & 156 \\
O-linked & 244 & 76 \\
\hline
\end{tabular}

glycosylation is generally associated with a $\mathrm{N}[! \mathrm{P}][\mathrm{ST}][\mathrm{P}]$ motif which is highly specific and aids in the predictor learning. Thus, we further classified the N-linked glycosylation sites into two subsets: a motif subset which contained all the glycosylation sites located in such motif and a non-motif set which did not include any obvious motifs. In addition, given that the O-linked glycosylation usually occurs on two different types of residues Serine (S) and Threonine $(\mathrm{T})$, we constructed two different models for each residue type separately. We then removed the redundant sequences from this dataset with the sequence identity of $30 \%$ by using the CD-HIT program. The statistical summary of this dataset is shown in Table 7.

We randomly split the dataset into the training sets and independent test sets with the ratio of 7 to 3 . The training sets were used for constructing the PA2DE (V2.0) model for the web server and the independent test sets were used for benchmarking the predictive performance with other existing methods. A statistical summary of the training set and independent test set is shown in Table 8.

We adopted the PU-learning protocol suggested in a recent work, MutPred2 [35] to re-train the PA2DE (V2.0) model based on the top 100 ranked features of the training set. The unlabelled dataset was first generated by randomly selecting 20 non-glycosylation sites from each glycosylated protein in the training data set. Then, the positive and unlabelled datasets were used to perform feature selection. The mRMR algorithm was employed to identify the top 100 ranked features for each type of glycosylation. The feature selection results are shown in Table 9.

For each type of glycosylation, a final unlabelled set was generated by further randomly selecting 10,000 non-glycosylation sites from the training set. In doing so, we ensure the estimation of class prior probability is fairly low $\left(1 \times 10^{-4}\right)$. As the glycosylation site prediction is a problem with class imbalance, the mislabelled samples exist in the unlabelled set with a relatively low fraction. For these types of glycosylation with fewer than 10,000 unlabelled samples, all the non-glycosylation sites were included. Then, a five-fold cross-validation test was performed on the training datasets. The summary of the training datasets for each type of glycosylation and the corresponding predictive performance are shown in Table 10.
In order to objectively evaluate the performance of our method, we compared the predictive performance of PA2DE (V2.0) with several state-of-the-art methods, including GlycoEP, NetNGlyc, NetOGlyc, and ModPred, on the independent test datasets. In order to perform the prediction, the protein sequences of the independent test datasets were submitted to the web servers/softwares of these methods with the default or recommended settings to obtain the prediction results, which were then used for evaluating the predictive performance of these methods. We randomly selected the equal number of non-glycosylation sites to the number of glycosylation sites as the negative samples from the glycosylated proteins in the independent test datasets. For example, if a protein sequence contained $n \mathrm{~N}$-linked glycosylation sites, we randomly selected $n$ amino acids (N) that were not labelled as N-linked glycosylation sites as the negative samples. The predictive performance for different types of glycosylation sites are shown in Table 11. We also generated the ROC curves (shown in Fig. 4) to evaluate and compare the performance of different methods.

As shown in Table 11 and Fig. 4, the performance comparison results indicate that for all five types of glycosylation, PA2DE (V2.0) achieved the best performance in terms of AUC. While for N-linked (non-motif) glycosylation, ModPred achieved the best ACC (0.807) and F1 score (0.773), while for O-linked (S) glycosylation, GlycoEP achieved the best F1 score (0.848).

Based on the trained models of PA2DE (V2.0), we further implemented an online web server that enables the users to predict potential novel glycosylation sites. The web server is freely available at http://glycomine.erc.monash.edu/Lab/GlycoMine_PU/, developed using Java Server Pages and managed by Tomcat 7 on a Linux server.

\section{Conclusions}

In this study, we have proposed a new computational method, PA2DE (V2.0), to address the task of protein glycosylation site prediction in the PU-learning scenario. A variety of algorithms, including supervised-learning (SVM and RF), PU-learning (PA2DE, PAODE, PNB, PTAN, and PFBC), and one-class classification algorithms (OneClassClassifiers and one-class SVMs) were extensively benchmarked, evaluated and compared with our proposed method in this study. Both benchmarking and independent tests showed that our proposed method achieved a competitive predictive performance compared with several supervised-learning algorithms for glycosylation-site prediction. Performance comparison results with the other existing methods indicate that the proposed method is capable of accurately predicting protein glycosylation sites. A publicly available web server has been implemented to facilitate the prediction of 


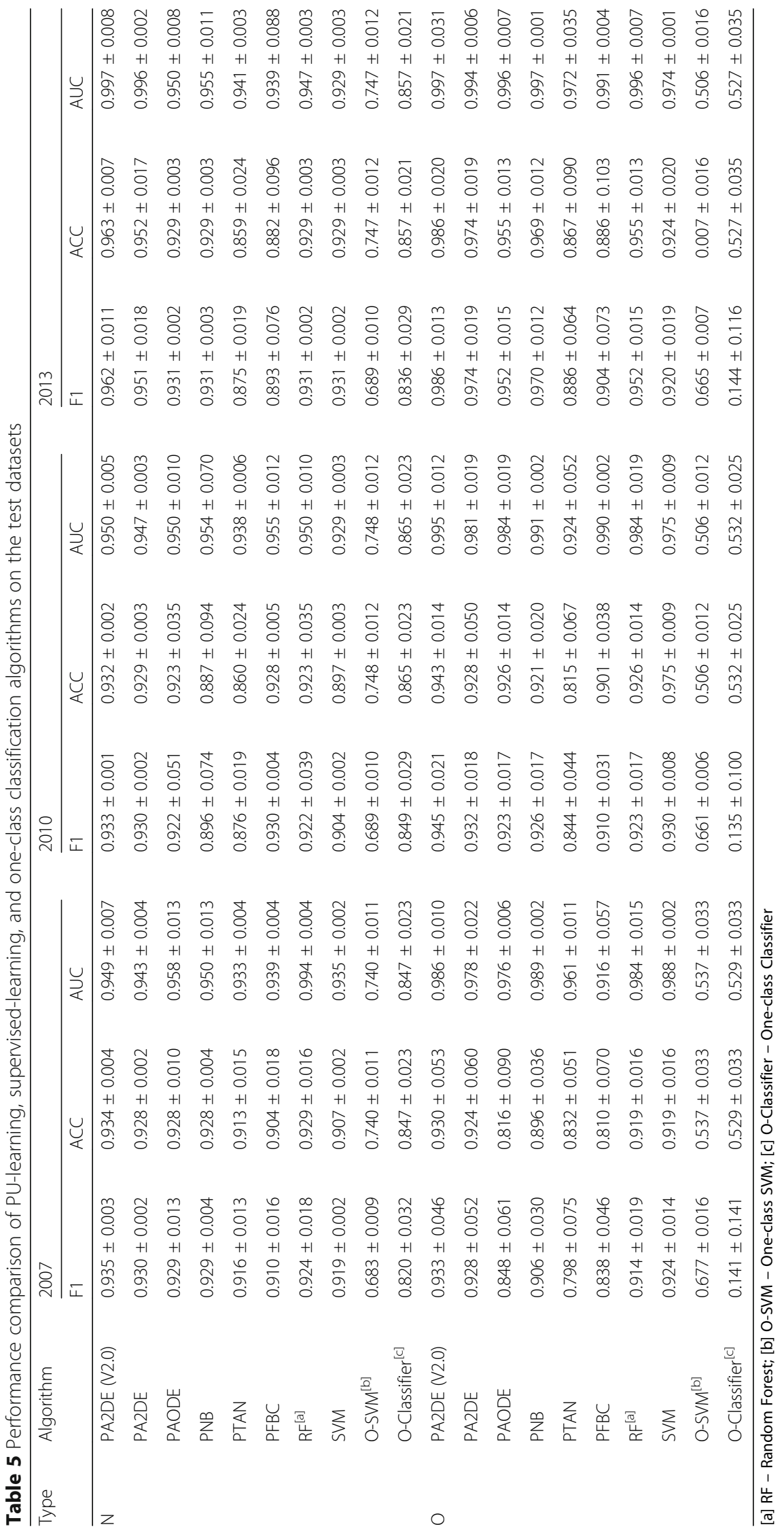


Table 6 Statistical significance of PA2DE performance in terms of F1 scores relative to the RF and SVM algorithms on the test datasets

\begin{tabular}{lllll}
\hline Type & Algorithm & 2007 & 2010 & 2013 \\
\hline N-linked & PA2DE & $6.35 \mathrm{E}-04$ & 0.0369 & $6.07 \mathrm{E}-23$ \\
& $\begin{array}{l}\text { Random Forest } \\
\text { PA2DE }\end{array}$ & $5.61 \mathrm{E}-21$ & $8.10 \mathrm{E}-06$ & $6.96 \mathrm{E}-23$ \\
& SVM & & & \\
& PA2DE (V2.0) & $2.44 \mathrm{E}-09$ & 0.0233 & $7.35 \mathrm{E}-40$ \\
& Random Forest & & & \\
& PA2DE (V2.0) & $1.09 \mathrm{E}-32$ & $1.34 \mathrm{E}-06$ & $8.33 \mathrm{E}-40$ \\
& SVM & & & \\
& PA2DE & 0.0104 & $6.10 \mathrm{E}-04$ & $1.86 \mathrm{E}-09$ \\
& Random Forest & & & \\
& PA2DE & 0.4566 & 0.0210 & $9.64 \mathrm{E}-16$ \\
& SVM & & & \\
& PA2DE (V2.0) & $6.23 \mathrm{E}-04$ & $1.04 \mathrm{E}-04$ & $6.53 \mathrm{E}-20$ \\
& Random Forest & & & \\
& PA2DE (V2.0) & 0.0986 & $9.19 \mathrm{E}-04$ & $8.19 \mathrm{E}-29$ \\
& SVM & & & \\
\hline
\end{tabular}

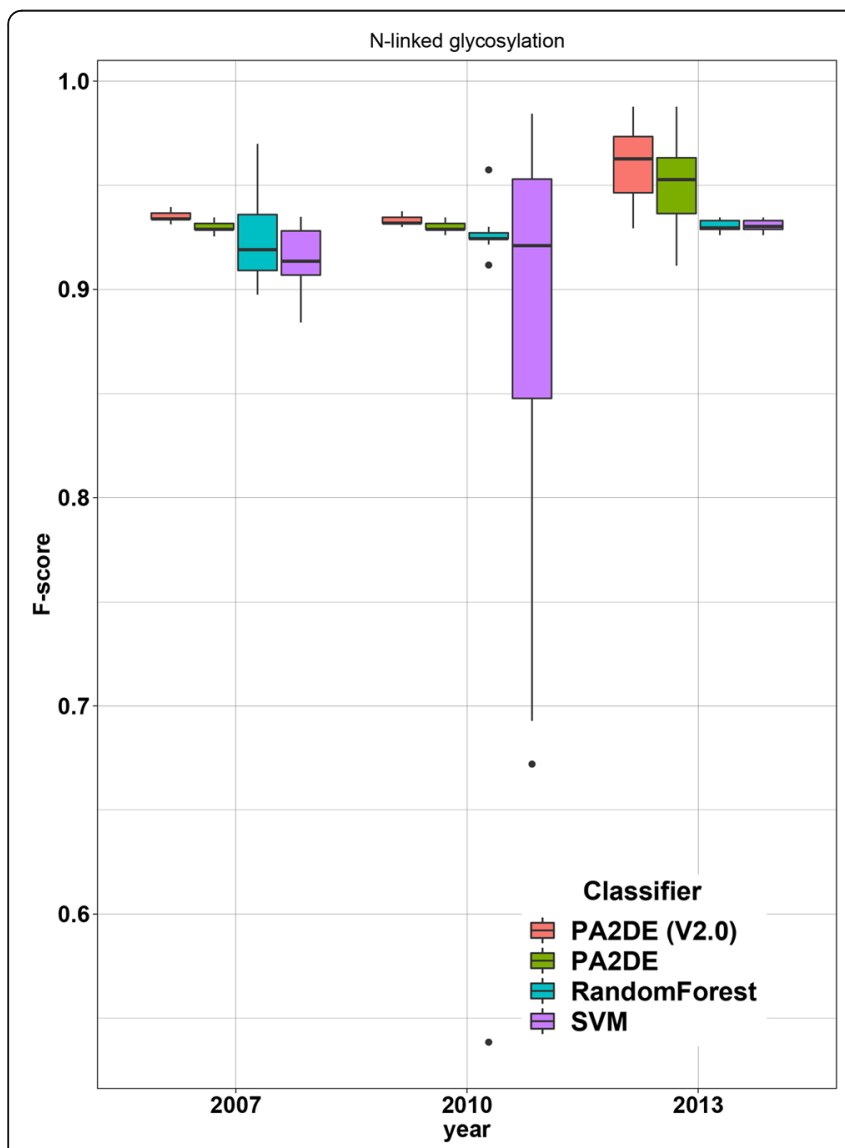

potential glycosylated protein substrates and glycosylation sites. With the success of applying PU-learning scheme to protein glycosylation prediction in this study, we anticipate that such methods can be widely employed to facilitate the prediction of other protein functional sites, including other different types of PTMs.

\section{Methods}

\section{Data collection and pre-processing}

As noted, four datasets comprising experimentally verified human C-, N-, and O-linked glycosylation sites from years 2007, 2010, 2013, and 2016 were collected from the UniProt database. To avoid overfitting and performance over-estimation, we removed sequence redundancy from all four datasets using the CD-HIT program [49] by setting the identity between any two sequences to $\leq 30 \%$.

The predictive performance of traditional supervisedlearning algorithms is contingent on the quality of both positive and negative samples. Similar to previous studies $[29,30]$, experimentally determined glycosylation sites were used as positive samples (Table 2). An increasing number of $\mathrm{C}$-, $\mathrm{N}$-, and $\mathrm{O}$-linked glycosylation sites and glycosylated proteins were identified from 2007 to 2016 (e.g., increasing from 1245 N-linked glycosylation

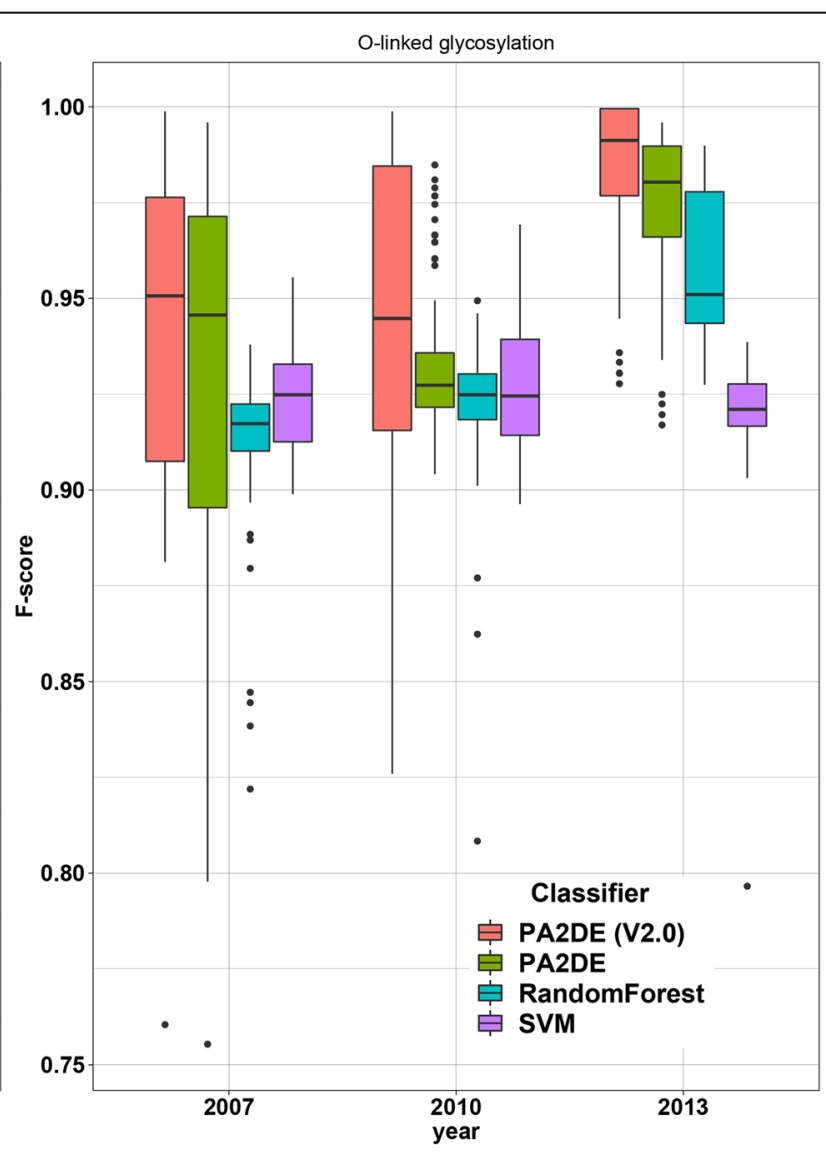

Fig. 3 Boxplots showing that PA2DE outperformed the RF and SVM algorithms in terms of F1 score on the test datasets 
Table 7 A statistical summary of glycosylated proteins and glycosylation sites collected from UniProt, dbPTM and PhosphoSitePlus

\begin{tabular}{|c|c|c|c|c|}
\hline \multirow[t]{2}{*}{ Type } & \multicolumn{2}{|c|}{ Before redundancy removal } & \multicolumn{2}{|c|}{ After redundancy removal } \\
\hline & Num. of Proteins & Num. of Sites & Num. of Proteins & Num. of Sites \\
\hline C-linked & 13 & 134 & 10 & 109 \\
\hline $\mathrm{N}$-linked (motif) & 1103 & 3850 & 770 & 2669 \\
\hline N-linked (non-motif) & 100 & 158 & 91 & 146 \\
\hline O-linked (S) & 192 & 683 & 165 & 602 \\
\hline O-linked (T) & 169 & 2150 & 155 & 2095 \\
\hline
\end{tabular}

sites in 2007 to 2805 in 2016). In-depth analysis of annotation changes in the data spanning these 4 years is provided in the section "Dataset analysis". Importantly, this indicated that a sizable number of non-glycosylation sites previously mislabelled and treated as negative sites (due to limitations in the experimental methods at the time) should be used as valid positive sites. Obviously, the inclusion of such mislabelled data will affect the performance evaluation of glycosylation-prediction models. Therefore, it is reasonable to assume that a portion of other experimentally unexplored residues, including tryptophan, asparagine, serine, and threonine, can be potentially identified as $\mathrm{C}$-, $\mathrm{N}$-, or O-linked glycosylation sites as experimental technologies continue to advance. Note that all current computational methods for glycosylation prediction were developed based on the labelling of positive and negative samples, which is consistent with traditional supervised-learning schemes. Therefore, it is difficult for existing methods to retrain or update the models in order to keep pace with rapidly updated data, especially concerning previously mislabelled negative samples. In this study, we predicted glycosylation sites by using a PU-learning scheme.

For benchmarking tests, we employed glycosylation sites retrieved from 2007, 2010, and 2013 as the positive samples used to train the classifiers. For traditional supervised-learning models (i.e., SVMs and RF), we randomly selected the same number of non-glycosylation sites as negative samples in order to construct the negative training datasets. As noted, such negative samples could be mislabelled due to limitations in experimental technologies. By contrast, for PU-learning models, such negative samples were treated as unlabelled samples. Because one-class learners only require information

Table 8 Numbers of glycosylation sites included in the training sets and independent test sets

\begin{tabular}{lll}
\hline Type & Training set & Independent test set \\
\hline C-linked & 76 & 33 \\
N-linked (motif) & 1869 & 800 \\
N-linked (non-motif) & 102 & 44 \\
O-linked (S) & 421 & 181 \\
O-linked (T) & 1467 & 628 \\
\hline
\end{tabular}

concerning the target class (i.e., glycosylation sites), there is no need to assign any negative or unlabelled samples for such models.

For the performance test set, we selected glycosylation sites that were experimentally annotated exclusively in 2016 as positive test samples. Negative samples (i.e., those having been consistently labelled as non-glycosylation sites across all 4 years) were randomly selected to constitute the negative dataset, with an equal number of positive samples used for each type of glycosylation. This random-sampling procedure was repeated 100 times. Due to insufficient test data for C-linked glycosylation (only three sites available), the performance test was constructed only for $\mathrm{N}$ - and O-linked glycosylation.

\section{Feature extraction and selection}

A local sliding window comprising 15 residues (i.e., seven upstream residues and seven downstream residues centred on the glycosylation site) [29] was used for feature extraction. This 15-residue peptide can be represented as [50]:

$$
\boldsymbol{P}=p_{1} p_{2} \ldots p_{8} \ldots p_{14} p_{15}
$$

where $p_{i}$ denotes the $i$-th residue of the peptide, $\boldsymbol{P}$, and $p_{8}$ denotes the glycosylation site. In this study, we extracted six groups of sequence-derived features to encode a peptide and train the machine-learning model. The first group consists of: 1) 20 amino acid compositions [51], and 2) 400 dipeptide amino acid compositions [52].

The second group includes three different types of autocorrelation features: 1) 240 normalized Moreau-Broto autocorrelation features [53, 54]; 2) 240 Moran autocorrelation features [55]; and 3) 240 Geary autocorrelation features [56]. The autocorrelation features measure the level of correlation between two peptide sequences according to their physicochemical properties.

The third group is 'Composition-Transition-Distribution (CTD)' [57], which includes three types of features: 1) 21 composition features, 2) 21 transition features, and 3) 105 distribution features. These features are calculated based on physicochemical properties that represent the amino acid-specific distribution of a specific structural or physicochemical property within a peptide. 
Table 9 The number of different selected feature groups as result of feature selection

\begin{tabular}{lllllll}
\hline Type & AAC & Auto-correlation & CTD & Sequence-order & Pseudo-AAC & AAindex \\
\hline C-linked & 3 & 23 & 2 & 4 & 5 & 63 \\
N-linked (motif) & 2 & 1 & 4 & 2 & 4 & 87 \\
N-linked (non-motif) & 4 & 1 & 3 & 2 & 4 & 36 \\
O-linked (S) & 11 & 7 & 13 & 3 & 2 & 2 \\
O-linked (T) & 8 & 4 & 8 & 2 & 63 \\
\hline
\end{tabular}

The fourth group includes two sequence-order-feature sets: 1) 60 sequence-order-coupling number features, and 2) 100 quasi-sequence-order features [58].

The fifth group contains two types of pseudo-aminoacid-composition features: 1) 50 type I features; and 2) 50 type II features [52].

The sixth group contains 8400 AAindex features extracted from the AAindex database [59].

A total of 9927 features were extracted and calculated. It is possible that such a high-dimensional feature set might contain certain noisy and irrelevant features, resulting in disfavorable model training and decreased predictive performance. To remove such features, we applied the mRMR (minimum Redundancy and Maximum Relevance) algorithm [40] and selected the top 100 features contributing the most to each $\mathrm{C}_{-}, \mathrm{O}_{-}$, and $\mathrm{N}$-linked glycosylation event. mRMR evaluates the relevance and redundancy of two features, $x$ and $y$, based on mutual information, which is defined as:

$$
I(x, y)=\iint p(x, y) \log \frac{p(x, y)}{p(x) p(y)} d x d y,
$$

where $p(x, y)$ is the joint probability of feature $x$ and $y$, where $p(x)$ and $p(y)$ are marginal probabilities.

\section{PU-learning algorithms}

Current PU-learning algorithms can be generally categorized into two main types. The first type has been implemented as a 'two-step' strategy, where the algorithms identify reliable negative samples from the unlabelled dataset first and then employ both the positive samples and these identified reliable negative samples to train a classifier in the second step. This procedure needs to be

Table 10 Summary of the training datasets and performance results of PA2DE (V2.0)

\begin{tabular}{|c|c|c|c|c|c|}
\hline \multirow[t]{2}{*}{ Type } & \multicolumn{2}{|c|}{ Number of Sites } & \multirow[t]{2}{*}{ AUC } & \multirow[t]{2}{*}{ ACC } & \multirow[t]{2}{*}{ F1 } \\
\hline & Positive & Unlabelled & & & \\
\hline C-linked & 76 & 258 & 0.997 & 0.981 & 0.981 \\
\hline $\mathrm{N}$-linked (motif) & 1869 & 7111 & 0.927 & 0.886 & 0.894 \\
\hline N-linked (non-motif) & 102 & 4888 & 0.874 & 0.974 & 0.973 \\
\hline O-linked (S) & 421 & 8331 & 0.974 & 0.972 & 0.972 \\
\hline O-linked (T) & 1467 & 10,000 & 0.876 & 0.857 & 0.859 \\
\hline
\end{tabular}

repeated until a certain threshold (e.g., a performance measure, such as Matthews's correlation coefficient or AUC) is achieved. To the best of our knowledge, this represents a predominant strategy currently practiced in bioinformatics research and has been adopted for identification and prediction of disease-associated genes from the human genome [60-62], protein pupylation prediction [63, 64], kinase substrates identification [65], protein subcellular localization prediction [66], and drug interactions prediction [67].

The second type focuses on evolving traditional supervised-learning algorithms to enable learning from both positive and unlabelled data. To date, several promising algorithms have been reported based on the evolution of classic supervised-learning algorithms, including decision trees (C4.5) [68] and Bayesian classifiers [69]. For example, POSC4.5 [70] was proposed based on the C4.5 algorithm, and based on Bayesian theory, He et al. [71]

Table 11 Performance comparison results between different methods on the independent test datasets

\begin{tabular}{lllll}
\hline Type & Methods & AUC & ACC & F1 \\
\hline C-linked & PA2DE (V2.0) & 0.999 & 0.983 & 0.984 \\
& GlycoEP & 0.546 & 0.600 & 0.647 \\
N-linked (motif) & ModPred & 0.933 & 0.933 & 0.938 \\
& PA2DE (V2.0) & 0.893 & 0.815 & 0.820 \\
& GlycoEP & 0.697 & 0.637 & 0.638 \\
& NetNGlyc & 0.638 & 0.616 & 0.627 \\
N-linked (non-motif) & ModPred & 0.837 & 0.782 & 0.791 \\
& PA2DE (V2.0) & 0.872 & 0.761 & 0.758 \\
& GlycoEP & 0.669 & 0.648 & 0.644 \\
& NetNGlyc & 0.630 & 0.716 & 0.675 \\
& ModPred & 0.842 & 0.807 & 0.773 \\
O-linked (S) & PA2DE (V2.0) & 0.915 & 0.859 & 0.766 \\
& GlycoEP & 0.796 & 0.787 & 0.848 \\
& ModPred & 0.873 & 0.770 & 0.670 \\
& NetOGlyc & 0.770 & 0.662 & 0.583 \\
& PA2DE (V2.0) & 0.864 & 0.793 & 0.827 \\
& GlycoEP & 0.739 & 0.694 & 0.747 \\
O-linked (T) & ModPred & 0.821 & 0.746 & 0.781 \\
& NetOGlyc & 0.769 & 0.718 & 0.763 \\
\hline
\end{tabular}




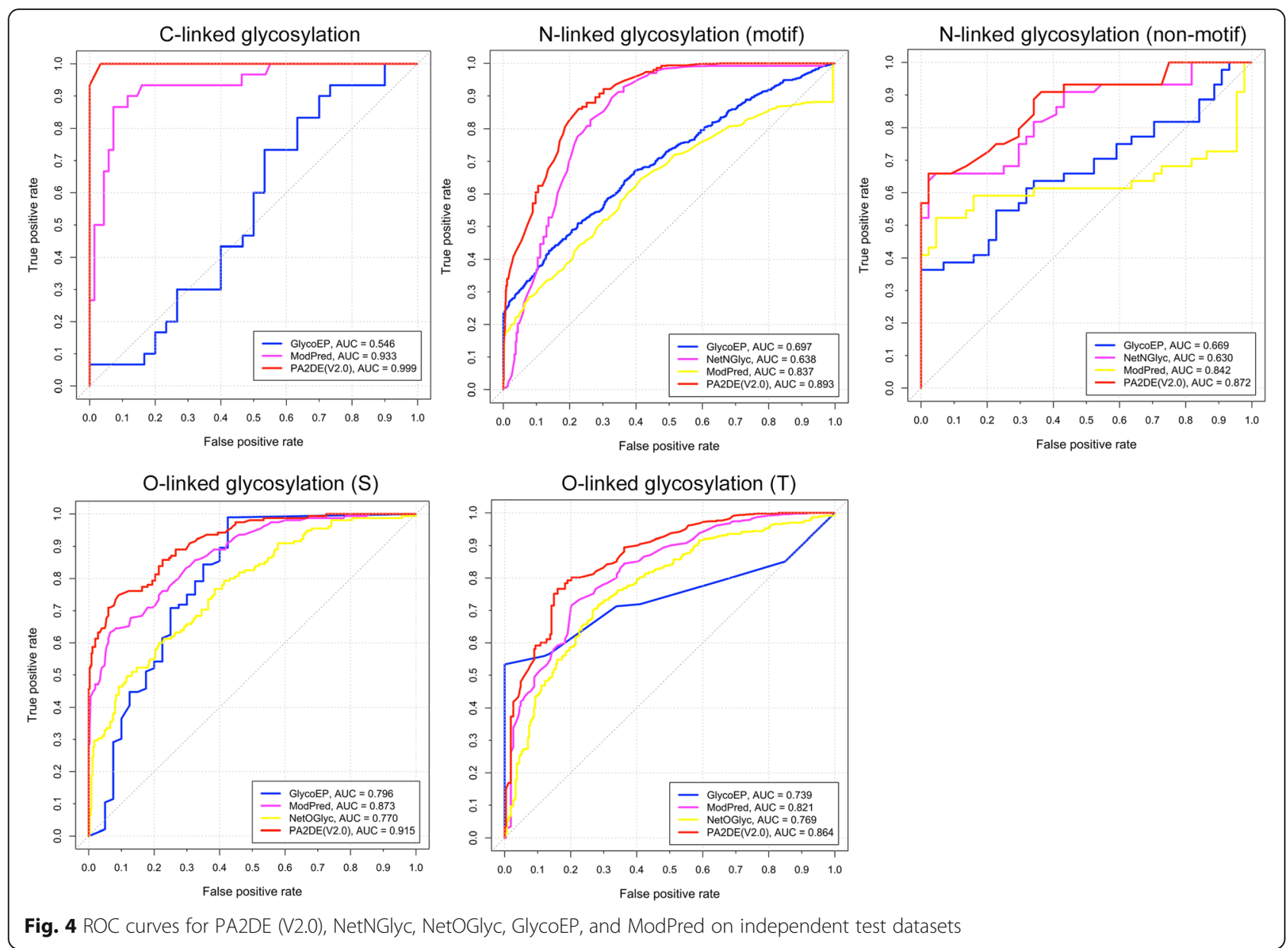

proposed a series of Bayesian classifiers for PU-learning, including PTAN (Positive Tree Augmented Naïve Bayes), PFBC (Positive Full Bayesian Network Classifier), PNB (Positive Naïve Bayes), and PAODE (Positive Averaged One-Dependence Estimators), according to the 'selected completely at random' assumption [36]. Previously, we proposed PA $n \mathrm{DE}$ (Positive Averaged $n$-Dependence Estimators) [72], which extends the AnDE algorithm [73] based on the 'selected completely at random' assumption.

$\mathrm{A} n \mathrm{DE}$ relaxes the attribute-independence assumption by selecting $n$ parent-attributes and assuming that all other attributes are independent of the given class label. The classification algorithm used by PAnDE for a sample, $\mathbf{x}$, is described as follows:

$$
\operatorname{PA} n \mathrm{DE}(\mathbf{x})=\arg \max _{y} \sum_{S \in\left(\begin{array}{c}
A \\
n
\end{array}\right)} \delta\left(x_{S}\right) P\left(y, x_{S}\right) \prod_{m=1}^{n} P\left(x_{m} \mid y, x_{S}\right),
$$

where $x_{m}$ denotes the value of attribute $X_{m},\left(\begin{array}{c}A \\ n\end{array}\right)$ denotes the set of all size- $n$ subsets of the attribute set $A=\{1, \ldots, k\}$, and $x_{S}$ denotes a tuple of parent attributes having $n$ attributes. The $\delta\left(x_{S}\right)$ function is used to avoid using parent attributes, the values of which do not occur in the training data, and $\delta\left(x_{S}\right)=1$ if $x_{S}$ occurs in the training dataset [otherwise $\delta\left(x_{S}\right)=0$ ]. Note that in the case that all $\delta\left(x_{S}\right)=0$, eq. (1) becomes:

$$
\operatorname{PA} n D E(\mathbf{x})=\operatorname{PA}(n-1) \operatorname{DE}(\mathbf{x}) .
$$

Empirical studies showed that PAnDE outperformed PNB and PAODE according to evaluation using $20 \mathrm{UCI}$ datasets and the protein glycosylation datasets collected in GlycoMine [72].

The original PAnDE algorithm applies the estimation method based on the 'selected completely at random' assumption to estimate the class priors, which has been shown to overestimate class priors, especially in cases where the true class priors are extremely small $[74,75]$. Recently, a very useful algorithm, termed AlphaMax [35, 74, 75], has been proposed to provide a new solution to estimate class priors. Considering that glycosylation prediction is a class imbalance problem and the mislabelled data in unlabelled set is low fraction, we thus used this new estimation method 
for class prior estimation in the PAnDE algorithm, referred to as PAnDE (V2.0).

Based on Bayes' theorem and the conditional independence assumption, PNB was initially devised based on a multinomial model of naïve Bayes (NB) for text classification [76]. This algorithm requires users to provide the prior probability of a positive class in order to estimate the probability for each class. Further, $\mathrm{He}$ et al. [71] extended PNB based on the 'selected completely at random' assumption in order to handle general classification tasks, with no requirement to provide prior probability.

PAODE [36] was proposed based on the AODE [77] algorithm (i.e., $\mathrm{A} 1 \mathrm{DE}$ ), which is a special version of $\mathrm{A} n \mathrm{DE}$ $(n=1)$ that relaxes the attribute independence assumption by using one super-parent attribute and considering all other attributes as conditionally independent, given this super-parent. Similarly, the proposed PAODE algorithm (i.e., PA1DE) is a special version of PA $n \mathrm{DE}$, where $n=1$.

PTAN is a another version of the tree-augmented NB (TAN) [78] algorithm for positive-unlabeled learning. The TAN algorithm approximates interactions between attributes by using a tree structure imposed upon the NB structure. TAN-structure learning occurs through computation of the conditional mutual information between two attributes, given a specific class label.

PFBC was proposed based on full Bayesian network classifier (FBC) [79], where the conditional probability table for each attribute is a decision tree. Learning an order of attributes is the most important process in constructing a full Bayesian network. The experimental results reported by $\mathrm{He}$ et al. [71] demonstrate that PFBC is more robust against unlabelled data.

In this study, we compared the predictive performance of our newly proposed PA2DE (V2.0) algorithm with the other five Bayesian PU-learning algorithms PA2DE, PNB, PAODE, PTAN, and PFBC for predicting glycosylation and further compared its predictive performance with several supervised-learning and one-class classification algorithms.

\section{Supervised-learning algorithms}

Two representative supervised-learning algorithms, RF and SVMs were used to compare the predictive performance of PU-learning and one-class classification algorithms. These two algorithms have been widely used to solve a variety of bioinformatics tasks and also in protein glycosylation prediction $[11,29,30,80-83]$ with the results providing outstanding predictive performance. In our study, we employed implementations of RF and SVM based on the WEKA machine-learning platform [84]. All corresponding parameters used for the two algorithms were set as the default values.

\section{One-class classification algorithms}

One-class classification algorithms identify samples of a specific class by learning from a training set containing samples only from this class. One-class classification has been widely applied in a variety of real-world scenarios, such as outlier [85] and novelty detection [86]. We also attempted to apply this learning method to glycosylation identification. We selected two state-of-the-art one-class classification algorithms implemented in WEKA (one-class SVMs [87] and OneClassClassifiers [88]) and used the same positive samples to train these one-class classifiers. A testing sample was predicted as ' 1 ' if the trained classifiers regarded the sample as positive; otherwise, it was predicted as '?'. We regarded samples predicted as '?' as predicted negative samples. Based on this strategy, we evaluated the performance of the one-class classifiers using the same performance measures as those for the supervised- and PU-learning methods.

\section{Performance evaluation}

Three performance measures were employed to evaluate the predictive performance of the supervised- and PU-learning schemes and facilitate comparisons between different methods, including AUC, F1, [36, 70] and ACC (Accuracy). These measurements are defined as follows:

$$
\begin{aligned}
& \mathrm{F} 1=\frac{2 \times \text { Precision } \times \text { Recall }}{\text { Precision }+ \text { Recall }}, \\
& \mathrm{ACC}=\frac{T P+T N}{T P+T N+F P+F N},
\end{aligned}
$$

where Precision and Recall in (5) are respectively defined as

$$
\begin{aligned}
& \text { Precision }=\frac{T P}{T P+F P}, \\
& \text { Recall }=\frac{T P}{T P+F N} .
\end{aligned}
$$

where $T P, T N, F P$, and $F N$ represent the numbers of true positives, true negatives, false positives, and false negatives, respectively.

\footnotetext{
Abbreviations

ACC: Accuracy; AUC: Area Under the ROC Curve; FN: False Negative; FP: False Positive; PAnDE: Positive Averaged $n$-Dependence Estimators; PAODE: Positive Averaged One-Dependence Estimators; PFBC: Positive Full Bayesian Network Classifier; PNB: Positive Naïve Bayes; PTAN: Positive Tree Augmented Naive Bayes; PTM: Post-Translational Modification; PUlearning: Positive-Unlabelled learning; RF: Random Forest; SVM: Support Vector Machine; TN: True Negative; TP: True Positive
}

\section{Acknowledgments}

We acknowledge the anonymous reviewers' constructive comments, which have greatly helped to improve the scientific quality of this study.

\section{Funding}

This research was supported by grants from the Australian Research Council (ARC) (LP110200333 and DP120104460), the National Health and Medical 
Research Council of Australia (NHMRC) (4909809, 1127948 and 1144652), the National Institute of Allergy and Infectious Diseases of the National Institutes of Health (R01 Al111965), and a Major Inter-Disciplinary Research (IDR) project awarded by Monash University. A.W.P. is currently supported by an NHMRC Principal Research Fellowship (1137739). C.L. is currently an NHMRC CJ Martin Early Career Research Fellow (1143366). T.L. is an ARC Australian Laureate Fellow (FL130100038). The funding body played any roles in the design of the study and collection, analysis, and interpretation of data and in writing the manuscript.

\section{Availability of data and materials}

The datasets of this study are available at http://glycomine.erc.monash.edu/ Lab/GlycoMine_PU/.

\section{Authors' contributions}

JS., CL. and FL. conceived the initial idea and designed the methodology. FL. implemented the experiments and processed the results. All authors drafted, revised and approved the final manuscript.

\section{Ethics approval and consent to participate}

Not applicable.

\section{Consent for publication}

Not applicable.

\section{Competing interests}

J.S. is an Associate Editor of BMC Bioinformatics.

\section{Publisher's Note}

Springer Nature remains neutral with regard to jurisdictional claims in published maps and institutional affiliations.

\section{Author details}

${ }^{1}$ Infection and Immunity Program, Biomedicine Discovery Institute and Department of Biochemistry and Molecular Biology, Monash University, Melbourne, VIC 3800, Australia. ${ }^{2}$ Monash Centre for Data Science, Faculty of Information Technology, Monash University, Melbourne, VIC 3800, Australia. ${ }^{3}$ College of Information Engineering, Northwest A and F University, Yangling 712100, Shaanxi, China. ${ }^{4}$ Gordon Life Science Institute, Boston, MA 02478, USA. ${ }^{5}$ Center for Informational Biology, School of Life Science and Technology, University of Electronic Science and Technology of China, Chengdu 610054, China. ${ }^{6}$ Infection and Immunity Program, Biomedicine Discovery Institute and Department of Microbiology, Monash University, Melbourne, VIC 3800, Australia. ${ }^{7}$ Department of Biology, Institute of Molecular Systems Biology, ETH Zürich, 8093 Zürich, Switzerland.

Received: 5 July 2018 Accepted: 22 February 2019 Published online: 06 March 2019

\section{References}

1. Moremen KW, Tiemeyer M, Nairn AV. Vertebrate protein glycosylation: diversity, synthesis and function. Nat Rev Mol Cell Biol. 2012;13(7):448-62.

2. Kristic J, Lauc G. Ubiquitous importance of protein glycosylation. Methods Mol Biol. 2017;1503:1-12.

3. Zhang $X$, Wang Y. Glycosylation quality control by the Golgi structure. J Mol Biol. 2016:428(16):3183-93.

4. Ohtsubo K, Marth JD. Glycosylation in cellular mechanisms of health and disease. Cell. 2006;126(5):855-67.

5. Dwek RA. Biological importance of glycosylation. Dev Biol Stand. 1998; 96:43-7.

6. Veillon L, Zhou S, Mechref Y. Quantitative Glycomics: a combined analytical and bioinformatics approach. Methods Enzymol. 2017;585:431-77.

7. Aoki-Kinoshita KF, Kanehisa M. Bioinformatics approaches in glycomics and drug discovery. Curr Opin Mol Ther. 2006;8(6):514-20.

8. von der Lieth CW, Bohne-Lang A, Lohmann KK, Frank M. Bioinformatics for glycomics: status, methods, requirements and perspectives. Brief Bioinform. 2004:5(2):164-78.

9. Pinho SS, Reis CA. Glycosylation in cancer: mechanisms and clinical implications. Nat Rev Cancer. 2015;15(9):540-55.

10. Xu C, Ng DT. Glycosylation-directed quality control of protein folding. Nat Rev Mol Cell Biol. 2015;16(12):742-52.
11. Bao W, Yuan C-A, Zhang Y, Han K, Nandi AK, Honig B, Ds H. Mutli-features Predction of protein translational modification sites. IEEE/ACM transactions on computational biology and bioinformatics. 2017.

12. Li F, Fan C, Marquez-Lago TT, Leier A, Revote J, Jia C, Zhu Y, Smith Al, Webb Gl, et al. PRISM: a comprehensive 3D structure database for posttranslational modifications and mutations with functional impact. bioRxiv. 2019:523308.

13. Neelofar K, Ahmad J. Glycosylation gap in patients with diabetes with chronic kidney disease and healthy participants: a comparative study. Indian J Endocrinol Metab. 2017;21(3):410-4.

14. Sadurni A, Kehr G, Ahlqvist M, Peilot Sjogren H, Kankkonen C, Knerr L, Gilmour R. Fluorine-directed glycosylation enables the Stereocontrolled synthesis of selective SGLT2 inhibitors for type II diabetes. Chemistry. 2017

15. Wolff SP, Dean RT. Glucose autoxidation and protein modification. The potential role of 'autoxidative glycosylation' in diabetes. Biochem J. 1987; 245(1):243-50.

16. Drabik A, Bodzon-Kulakowska A, Suder P, Silberring J, Kulig J, Sierzega M. Glycosylation changes in serum proteins identify patients with pancreatic Cancer. J Proteome Res. 2017;16(4):1436-44.

17. Ferreira JA, Magalhaes A, Gomes J, Peixoto A, Gaiteiro C, Fernandes E, Santos LL, Reis CA. Protein glycosylation in gastric and colorectal cancers: toward cancer detection and targeted therapeutics. Cancer Lett. 2017;387: 32-45.

18. Magalhaes A, Duarte HO, Reis CA. Aberrant glycosylation in Cancer: a novel molecular mechanism controlling metastasis. Cancer Cell. 2017;31(6):733-5.

19. Oliveira-Ferrer L, Legler K, Milde-Langosch K. Role of protein glycosylation in cancer metastasis. Semin Cancer Biol. 2017:44:141-52.

20. Roberts JD, Klein JL, Palmantier R, Dhume ST, George MD, Olden K. The role of protein glycosylation inhibitors in the prevention of metastasis and therapy of cancer. Cancer Detect Prev. 1998;22(5):455-62.

21. Steentoft C, Vakhrushev SY, Joshi HJ, Kong Y, Vester-Christensen MB, Schjoldager KT, Lavrsen K, Dabelsteen S, Pedersen NB, Marcos-Silva L, et al. Precision mapping of the human O-GaINAc glycoproteome through SimpleCell technology. EMBO J. 2013;32(10):1478-88.

22. Vergroesen RD, Slot LM, Hafkenscheid L, Koning MT, Scherer HU, Toes REM. Response to: 'Acquiring new N-glycosylation sites in variable regions of immunoglobulin genes by somatic hypermutation is a common feature of autoimmune diseases' by Visser et al. Ann Rheum Dis. 2017.

23. Visser A, Hamza N, Kroese FGM, Bos NA: Acquiring new N-glycosylation sites in variable regions of immunoglobulin genes by somatic hypermutation is a common feature of autoimmune diseases. Ann Rheum Dis 2017.

24. Gupta R, Brunak S. Prediction of glycosylation across the human proteome and the correlation to protein function. Pac Symp Biocomput. 2002:310-22.

25. Caragea C, Sinapov J, Silvescu A, Dobbs D, Honavar V. Glycosylation site prediction using ensembles of support vector machine classifiers. BMC Bioinformatics. 2007;8:438.

26. Hamby SE, Hirst JD. Prediction of glycosylation sites using random forests. BMC Bioinformatics. 2008:9:500.

27. Chauhan JS, Rao A, Raghava GP. In silico platform for prediction of N-, Oand C-glycosites in eukaryotic protein sequences. PLoS One. 2013;8(6): e67008.

28. Pejaver V, Hsu WL, Xin FX, Dunker AK, Uversky VN, Radivojac P. The structural and functional signatures of proteins that undergo multiple events of post-translational modification. Protein Sci. 2014;23(8):1077-93.

29. Li F, Li C, Wang M, Webb Gl, Zhang Y, Whisstock JC, Song J. GlycoMine: a machine learning-based approach for predicting $\mathrm{N}$-, C- and O-linked glycosylation in the human proteome. Bioinformatics. 2015;31(9):1411-9.

30. Li F, Li C, Revote J, Zhang Y, Webb Gl, Li J, Song J, Lithgow T. GlycoMine(struct): a new bioinformatics tool for highly accurate mapping of the human $\mathrm{N}$-linked and O-linked glycoproteomes by incorporating structural features. Sci Rep. 2016;6:34595.

31. De Comité F, Denis F, Gilleron R, Letouzey F: Positive and unlabeled examples help learning. In: Algorithmic Learning Theory: 1999. Springer: 219-230.

32. Niu G, du Plessis MC, Sakai T, Ma Y, Sugiyama M: Theoretical comparisons of positive-unlabeled learning against positive-negative learning. In: Advances in neural information processing systems: 2016. 1199-1207.

33. Menon A, Rooyen BV, Ong CS, Williamson B: Learning from Corrupted Binary Labels via Class-Probability Estimation. In: Proceedings of the 32nd International Conference on Machine Learning; Proceedings of Machine Learning Research: Edited by Francis B, David B. PMLR 2015: 125--134. 
34. Jain S, White M, Radivojac P: Recovering true classifier performance in positive-unlabeled learning. In: AAAl: 2017. 2066-2072.

35. Pejaver V, Urresti J, Lugo-Martinez J, Pagel KA, Lin GN, Nam H-J, Mort M, Cooper DN, Sebat J, lakoucheva LM, et al. MutPred2: inferring the molecular and phenotypic impact of amino acid variants. bioRxiv:2017.

36. Elkan C, Noto K: Learning classifiers from only positive and unlabeled data In: Proceedings of the 14th ACM SIGKDD international conference on Knowledge discovery and data mining: 2008. ACM: 213-220.

37. Chang S, Zhang Y, Tang J, Yin D, Chang Y, Hasegawa-Johnson MA, Huang TS: Positive-unlabeled learning in streaming networks. In: Proceedings of the 22nd ACM SIGKDD international conference on Knowledge discovery and data mining: 2016. ACM: 755-764.

38. Xu YY, Yang F, Zhang Y, Shen HB. Bioimaging-based detection of mislocalized proteins in human cancers by semi-supervised learning Bioinformatics. 2015;31(7):1111-9.

39. The UniProt C. UniProt: the universal protein knowledgebase. Nucleic Acids Res. 2017:45(D1):D158-69.

40. Peng HC, Long FH, Ding C. Feature selection based on mutual information: criteria of max-dependency, max-relevance, and min-redundancy. leee $T$ Pattern Anal. 2005;27(8):1226-38.

41. Li F, Li C, Marquez-Lago TT, Leier A, Akutsu T, Purcell AW, Smith Al, Lithgow T, Daly RJ, Song J, et al. Quokka: a comprehensive tool for rapid and accurate prediction of kinase family-specific phosphorylation sites in the human proteome. Bioinformatics. 2018.

42. Chen Z, Liu X, Li F, Li C, Marquez-Lago T, Leier A, Akutsu T, Webb Gl, $\mathrm{Xu} \mathrm{D}$, Smith Al, et al. Large-scale comparative assessment of computational predictors for lysine post-translational modification sites. Brief Bioinform. 2018.

43. Song J, Li F, Leier A, Marquez-Lago TT, Akutsu T, Haffari G, Chou KC, Webb Gl, Pike RN, Hancock J. PROSPERous: high-throughput prediction of substrate cleavage sites for 90 proteases with improved accuracy. Bioinformatics. 2018;34(4):684-7.

44. Song J, Wang Y, Li F, Akutsu T, Rawlings ND, Webb Gl, Chou K-C: iProt-Sub: a comprehensive package for accurately mapping and predicting proteasespecific substrates and cleavage sites. Briefings in Bioinformatics 2018: bby028-bby028.

45. Li F, Wang Y, Li C, Marquez-Lago TT, Leier A, Rawlings ND, Haffari G, Revote J, Akutsu T, Chou K-C et al: Twenty years of bioinformatics research for protease-specific substrate and cleavage site prediction: a comprehensive revisit and benchmarking of existing methods. Briefings in Bioinformatics 2018:bby077-bby077.

46. Fanchi M, Insung N, Lukasz K, Uversky VN. Compartmentalization and functionality of nuclear disorder: intrinsic disorder and protein-protein interactions in intra-nuclear compartments. Int J Mol Sci. 2015;17(1):24

47. Huang KY, Su MG, Kao HJ, Hsieh YC, Jhong JH, Cheng KH, Huang HD, Lee TY. dbPTM 2016: 10-year anniversary of a resource for post-translational modification of proteins. Nucleic Acids Res. 2016:44(D1):D435-46.

48. Hornbeck PV, Zhang B, Murray B, Kornhauser JM, Latham V, Skrzypek E. PhosphoSitePlus, 2014: mutations, PTMs and recalibrations. Nucleic Acids Res. 2015;43(Database issue):D512-20.

49. Fu L, Niu B, Zhu Z, Wu S, Li W. CD-HIT: accelerated for clustering the nextgeneration sequencing data. Bioinformatics. 2012;28(23):3150-2.

50. Cheng X, Zhao S-G, Lin W-Z, Xiao X, Chou K-C. pLoc-mAnimal: predict subcellular localization of animal proteins with both single and multiple sites. Bioinformatics. 2017;33(22):3524-31.

51. Nakashima H, Nishikawa K, Ooi T. The folding type of a protein is relevant to the amino acid composition. J Biochem. 1986;99(1):153-62.

52. Chou KC. Prediction of protein cellular attributes using pseudo-amino acid composition. Proteins: Structure, Function, and Bioinformatics. 2001;43(3): 246-55.

53. Feng Z-P, Zhang C-T. Prediction of membrane protein types based on the hydrophobic index of amino acids. J Protein Chem. 2000;19(4):269-75.

54. Chen Z, Zhao P, Li F, Leier A, Marquez-Lago TT, Wang Y, Webb Gl, Smith Al, Daly RJ, Chou KC, et al. iFeature: a python package and web server for features extraction and selection from protein and peptide sequences. Bioinformatics. 2018.

55. Horne DS. Prediction of protein helix content from an autocorrelation analysis of sequence hydrophobicities. Biopolymers. 1988;27(3):451-77.

56. Sokal RR, Thomson BA. Population structure inferred by local spatial autocorrelation: an example from an Amerindian tribal population. Am J Phys Anthropol. 2006;129(1):121-31.
57. Dubchak I, Muchnik I, Mayor C, Dralyuk I, Kim SH. Recognition of a protein fold in the context of the SCOP classification. Proteins: Structure, Function, and Bioinformatics. 1999;35(4):401-7.

58. Chou K-C. Prediction of protein subcellular locations by incorporating quasisequence-order effect. Biochem Biophys Res Commun. 2000;278(2):477-83.

59. Kawashima S, Pokarowski P, Pokarowska M, Kolinski A, Katayama T, Kanehisa M. AAindex: amino acid index database, progress report 2008. Nucleic Acids Res. 2008:36(Database issue):D202-5.

60. Jowkar GH, Mansoori EG. Perceptron ensemble of graph-based positiveunlabeled learning for disease gene identification. Comput Biol Chem. 2016; 64:263-70.

61. Yang P, Li X, Chua HN, Kwoh CK, Ng SK. Ensemble positive unlabeled learning for disease gene identification. PLoS One. 2014;9(5):e97079.

62. Yang P, Li XL, Mei JP, Kwoh CK, Ng SK. Positive-unlabeled learning for disease gene identification. Bioinformatics. 2012;28(20):2640-7.

63. Jiang M, Cao JZ. Positive-unlabeled learning for Pupylation sites prediction. Biomed Res Int. 2016;2016:4525786.

64. Nan X, Bao L, Zhao X, Zhao X, Sangaiah AK, Wang GG, Ma Z. EPuL: An Enhanced Positive-Unlabeled Learning Algorithm for the Prediction of Pupylation Sites. Molecules. 2017;(9):22.

65. Yang P, Humphrey SJ, James DE, Yang YH, Jothi R. Positive-unlabeled ensemble learning for kinase substrate prediction from dynamic phosphoproteomics data. Bioinformatics. 2016;32(2):252-9.

66. Xu Y-Y, Yang F, Shen H-B. Incorporating organelle correlations into semisupervised learning for protein subcellular localization prediction. Bioinformatics. 2016:32(14):2184-92.

67. Hameed PN, Verspoor K, Kusljic S, Halgamuge S. Positive-unlabeled learning for inferring drug interactions based on heterogeneous attributes. BMC Bioinformatics. 2017;18(1):140.

68. Quinlan JR: C4. 5: programs for machine learning: Elsevier; 2014.

69. Langley P, Iba W, Thompson K: An analysis of Bayesian classifiers. In: Aaai: 1992. 223-228.

70. Denis F, Gilleron R, Letouzey F. Learning from positive and unlabeled examples. Theor Comput Sci. 2005:348(1):70-83.

71. He J, Zhang Y, Li X, Wang Y: Bayesian classifiers for positive unlabeled learning. In: International Conference on Web-Age Information Management: 2011. Springer: 81-93.

72. Li F, Song J, Li C, Akutsu T, Zhang Y: PAnDE: Averaged n-Dependence Estimators for Positive Unlabeled Learning. ICIC Express Letters Part B: Applications, 8(9):11.

73. Webb Gl, Boughton JR, Zheng F, Ting KM, Salem H. Learning by extrapolation from marginal to full-multivariate probability distributions: decreasingly naive Bayesian classification. Mach Learn. 2012;86(2):233-72.

74. Jain S, White M, Trosset MW, Radivojac P: Nonparametric semi-supervised learning of class proportions. arXiv preprint arXiv:160101944 2016

75. Jain S, White M, Radivojac P: Estimating the class prior and posterior from noisy positives and unlabeled data. In: Advances in Neural Information Processing Systems: 2016. 2693-2701

76. Denis F, Laurent A, Gilleron R, Tommasi M: Text classification and co-training from positive and unlabeled examples. In: Proceedings of the ICML 2003 workshop: the continuum from labeled to unlabeled data: 2003. 80-87.

77. Webb Gl, Pazzani MJ: Adjusted probability naive Bayesian induction. In: Australian Joint Conference on Artificial Intelligence: 1998. Springer: 285295.

78. Friedman N, Geiger D, Goldszmidt M. Bayesian network classifiers. Mach Learn. 1997:29(2-3):131-63.

79. Su J, Zhang H: Full Bayesian network classifiers. In: Proceedings of the 23rd international conference on Machine learning: 2006. ACM: 897-904.

80. Xie HL, Fu L, Nie XD. Using ensemble SVM to identify human GPCRs Nlinked glycosylation sites based on the general form of Chou's PseAAC. Protein Eng Des Sel. 2013;26(11):735-42.

81. Song J, Li F, Takemoto K, Haffari G, Akutsu T, Chou KC, Webb Gl. PREvalL, an integrative approach for inferring catalytic residues using sequence, structural, and network features in a machine-learning framework. J Theor Biol. 2018;443:125-37.

82. Wei L, Hu J, Li F, Song J, Su R, Zou Q. Comparative analysis and prediction of quorum-sensing peptides using feature representation learning and machine learning algorithms. Brief Bioinform. 2018.

83. Zhang M, Li F, Marquez-Lago TT, Leier A, Fan C, Kwoh CK, Chou KC, Song J, Jia C. MULTiPly: a novel multi-layer predictor for discovering general and specific types of promoters. Bioinformatics. 2019. 
84. Witten IH, Frank E, Hall MA, Pal CJ: Data mining: practical machine learning tools and techniques: Morgan Kaufmann; 2016.

85. Abe N, Zadrozny B, Langford J: Outlier detection by active learning. In: Proceedings of the 12th ACM SIGKDD international conference on Knowledge discovery and data mining: 2006. ACM: 504-509.

86. Sebert DM. Outliers in statistical data. J Qual Technol. 1997;29(2):230

87. Manevitz LM, Yousef M. One-class SVMs for document classification. J Mach Learn Res. 2001;2(Dec):139-54.

88. Hempstalk K, Frank E, Witten IH: One-class classification by combining density and class probability estimation. In: Joint European Conference on Machine Learning and Knowledge Discovery in Databases: 2008. Springer: 505-519.

Ready to submit your research? Choose BMC and benefit from:

- fast, convenient online submission

- thorough peer review by experienced researchers in your field

- rapid publication on acceptance

- support for research data, including large and complex data types

- gold Open Access which fosters wider collaboration and increased citations

- maximum visibility for your research: over $100 \mathrm{M}$ website views per year

At $\mathrm{BMC}$, research is always in progress.

Learn more biomedcentral.com/submissions 